\title{
Prevalência de Alterações no Exame Citológico do Colo do Útero em Pacientes com Lúpus Eritematoso Sistêmico
}

\section{Prevalence of Abnormal Pap Smears in Patients with Systemic Lupus Erythematosus}

\author{
Bernardo Rodi Carvalho Barros ${ }^{(1)}$, Roberta Matschinske( ${ }^{(1)}$, Marília Barreto Silva ${ }^{(2)}$, Thelma Larocca Skare ${ }^{(2)}$
}

\section{RESUMO}

Introdução: estudos sugerem que mulheres com lúpus eritematoso sistêmico (LES) apresentam maior taxa de anormalidades no exame citológico do esfregaço cervical quando comparadas com a população geral. Objetivo: estudar a prevalência de anormalidades no exame citológico de Papanicolaou de pacientes com LES, procurando correlacionar o seu aparecimento com tempo de doença, uso de medicamentos imunossupressores e perfil de auto-anticorpos. Método: foram analisados 76 exames citológicos de pacientes do sexo feminino, portadoras de LES, e de 80 mulheres saudáveis. Nas pacientes com lúpus, foram verificados: tempo de doença, uso de anticoncepcionais, uso anterior de citostáticos (ciclofosfamida, azatioprina e metotrexato), antimaláricos e presença dos anticorpos anti-RO, anti-LA, anti-DNA. Resultado: a prevalência de alterações do Papanicolaou foi maior no grupo das pacientes com LES quando comparada com controles $(9,4 \%$ versus $1,2 \%, p=0,03)$. Não se encontrou associação entre o aparecimento de Papanicolaou alterado e o uso de ciclofosfamida $(\mathrm{p}=1,0)$, metotrexato $(\mathrm{p}=0,58)$, azatioprina $(\mathrm{p}=1,0)$, antimaláricos $(\mathrm{p}=0,05 \mathrm{l})$, tempo de doença $(\mathrm{p}=0,85)$, exposição, nem com perfil de auto-anticorpos estudado. Conclusão: existe maior prevalência de alterações do exame citológico de esfregaço vaginal em pacientes com LES na população estudada, a qual não depende do tempo da doença, uso de agentes imunossupressores e presença de anti-RO, anti-LA ou anti-DNA.

Palavras-chave: lúpus eritematoso sistêmico, esfregaço vaginal, imunossupressores.

\section{INTRODUÇÃO}

Existe uma associação interessante entre doenças de auto-imunidade e doenças malignas ${ }^{(1)}$. Algumas delas são bem reconhecidas como a ocorrência de linfomas nãoHodgkin em pacientes com síndrome de Sjögren ou artrite reumatóide e o aumento de neoplasias pulmonares em

\begin{abstract}
Background: studies have suggested that patients with systemic lupus erythematosus (SLE) have increased risk of developing abnormal results in Pap smears, when compared to the general population. Objective: to check the prevalence of abnormal Pap smears in patients with SLE compared to the general population relating it with disease duration, use of immunosuppressive and antimalarial agents and autoantibody profile. Method: we studied Pap smears of 76 patients with SLE and 80 healthy controls. In patients with SLE we assessed disease duration, use of cyclophosphamide, methotrexate, azathioprine, antimalarials and the presence of anti-RO, anti-LA and anti-DNA. Result: the prevalence of abnormal Pap smears was higher in the SLE group than controls $(9.4 \%$ versus $1.2 \%, p=0.03)$. No relationship was found between abnormal Pap smears and cyclophosphamide $(p=1.0)$, methotrexate $(p=0.58)$, azathioprine $(p=1.0)$ or antimalarial use $(p=0.051)$, disease duration $(p=0.85)$, neither with any of the studied auto antibodies. Conclusion: there is a higher prevalence of abnormal Pap smears in the studied SLE population than in normal people that is independent of the duration of the disease, use of immunosupressive medication or presence of autoantibodies.
\end{abstract}

Keywords: systemic lupus erythematosus, vaginal smears, immunosuppressive agents.

pacientes com esclerose sistêmica progressiva que sofrem de doença pulmonar intersticial ${ }^{(1)}$.

Também no lúpus eritematoso sistêmico (LES) esta associação tem sido sugerida. Um estudo longitudinal finlandês, de 205 pacientes, mostrou um risco relativo para desenvolvimento de câncer de 2,6 $6^{(2)}$; um outro, americano, envolvendo 606 mulheres pontuou esse risco em $2,0^{(3)}$. Um

Recebido em 29/01/07. Aprovado, após revisão, em 12/07/07. Declaramos a inexistência de conflitos de interesse.

Trabalho aprovado pelo Comitê de Ética: protocolo 1095/2005 - CE da Sociedade Evangélica Beneficente de Curitiba e realizado no Serviço de Reumatologia do Hospital Universitário Evangélico de Curitiba (PR).

1. Aluno(a) do $6^{\circ}$ ano do Curso de Medicina da Faculdade Evangélica do Paraná (Fepar).

2. Professora-assistente da Disciplina de Reumatologia da Fepar.

Endereço para correspondência: Thelma Larocca Skare, Rua João Alencar Guimarães, 796, CEP 80310-420 Curitiba, PR, e-mail: tskare@onda.com.br 
terceiro estudo, também americano, com 209 pacientes mostra um risco relativo de $1,4^{(4)}$.

Segundo Ramsey-Goldman et al. ${ }^{(3)}$, as neoplasias mais comumente encontradas no LES são linfomas, câncer de pulmão, de mama, de cérvix e carcinoma de canal anal. Todavia, nem todos os autores concordam com tais achados. Sultan et al..$^{(1)}$ e Abu Shakra et al. ${ }^{(5)}$ encontraram apenas aumento das neoplasias hematológicas.

Várias hipóteses pretendem justificar uma maior incidência de neoplasias em pacientes com LES. Entre elas, encontram-se: (a) anormalidades intrínsecas do sistema imunológico, decorrentes da própria doença, prejudicando a vigilância antitumoral; (b) presença de doenças associadas ao LES como a síndrome de Sjögren; (c) fatores geográficos, étnicos e raciais comuns a neoplasias e ao LES; (d) medicações utilizadas no tratamento do LES como azatioprina, metotrexato e ciclofosfamida; (e) interações entre as medicações e exposições virais ${ }^{(1,6-9)}$.

Neoplasias de colo uterino incidem em 20/100.000 mulheres nas Regiões Sul e Sudeste do Brasil ${ }^{(10)}$. Lesões malignas (e pré-malignas) de cérvix uterina fazem parte dos tumores descritos como sendo mais prevalentes no LES $^{(6,8,9)}$. Esse aspecto pretende ser explorado no presente estudo que tem por objetivo estabelecer a freqüência de alterações no esfregaço citopatológico (Papanicolaou) em uma população local de pacientes com LES e verificar se tal aumento está associado ao uso de medicações, tempo de doença e perfil de auto-anticorpos apresentado.

\section{PACIENTES E MÉTODOS}

Este estudo foi aprovado pelo Comitê de Ética local, sendo obtido consentimento livre e esclarecido das participantes.

Trata-se de um estudo observacional, controlado, incluindo 76 pacientes do sexo feminino com pelo menos quatro dos critérios classificatórios do American College of Rheumatology para diagnóstico de LES ${ }^{(11)}$ acompanhadas no Ambulatório de Reumatologia durante o segundo semestre de 2005 e o primeiro semestre de 2006. Foram consideradas elegíveis para o estudo todas as pacientes com vida sexual ativa, sendo excluídas pacientes histerectomizadas e menopausadas. A amostra obtida foi composta por todas as pacientes que freqüentaram o ambulatório de reumatologia durante o período anteriormente citado e que concordaram em participar do estudo.

As pacientes foram questionadas no próprio ambulatório sobre a realização de seu último exame citológico do colo uterino (exame de Papanicolaou), sendo solicitado que trouxessem o resultado em sua consulta seguinte. Caso a paciente em questão não tivesse realizado o exame havia mais de um ano da data da referida consulta, era oferecido um encaminhamento médico à Unidade Básica de Saúde para que fizesse a coleta de tal exame.

Foram estudados, retrospectivamente, por meio de registros no prontuário médico, os seguintes dados das pacientes incluídas no estudo: idade, data do diagnóstico do LES, tabagismo, uso de anticoncepcionais, presença de anticorpos anti-RO, anti-LA, anti-DNA, uso de drogas citotóxicas e cloroquina para tratamento do LES.

Os exames citológicos foram agrupados seguindo a classificação de Bethesda ${ }^{(12)}$. Esta segue as normativas do Instituto Nacional Americano do Câncer de 1988, modificado em 1991 e 2001, sendo feitas da seguinte forma:

1. Presença de células típicas: exame considerado negativo para malignidade.

2. Presença de células atípicas:

2a. Epitélio estratificado: LSIL ou LGSIL (lesão intra-epitelial escamosa de baixa malignidade); e HSIL ou HGSIL (lesão intra-epitelial escamosa de alto grau de malignidade);

2b. Epitélio glandular: presença de células endocervicais ou glandulares atípicas.

3. Presença de células de significado indeterminado:

3a. Epitélio estratificado: ASCUS (células do epitélio estratificado com significado indeterminado);

3b. Epitélio glandular: AGUS (células do epitélio glandular com significado indeterminado).

4. Presença de câncer:

4a. Epitélio estratificado: carcinoma escamoso.

4b. Epitélio glandular: adenocarcinoma endocervical in situ e adenocarcinoma (endocervical, endometrial, extra-uterino, não especificado) $)^{(11)}$.

As amostras de lesões de baixo grau (LSIL) correspondem à NIC (NIC = neoplasia intra-epitelial cervical) I, assim como as lesões de alto grau (HSIL) e carcinoma in situ são correspondentes às classificadas como NIC II, III ${ }^{(12)}$.

Para controle foram analisados 80 resultados de exames citopatológicos, provenientes de usuárias da Unidade Básica de Saúde Nossa Senhora Aparecida, localizada no Bairro Novo, em Curitiba (PR), obtidos durante um único mês e pareados para idade e atividade sexual. 


\section{ANÁLISE ESTATÍSTICA}

Os dados foram estudados por tabelas de freqüência e contingência, sendo usados os testes do qui-quadrado e Fisher para análise de associações de variáveis discretas e de Mann-Whitney para testes de correlação de variáveis contínuas, com auxílio do software Graph Pad Prism 4.0. O nível de significância adotado foi de $5 \%$.

\section{RESULTADOS}

$\mathrm{Na}$ amostra de 76 pacientes com LES, a média de critérios classificatórios para LES do American College of Rheumatology foi de $5 \pm 1,2$, existindo $36,8 \%(\mathrm{n}=28)$ pacientes com lesão renal. Ainda neste grupo, $10,5 \%(\mathrm{n}=8)$ pacientes estavam em uso de metotrexato, $23,5 \%(\mathrm{n}=18)$ haviam feito uso de ciclofosfamida, $15,7 \%(\mathrm{n}=12)$, de azatioprina, $1,3 \%(\mathrm{n}=1)$, de mofetil micofenolato, $76,3 \%(\mathrm{n}=58)$, de cloroquina e $85,5 \%(n=65)$, de glicocorticóides. Uma única paciente da amostra havia sido tratada exclusivamente de glicocorticóides e cerca de 38,1\% $(\mathrm{n}=29)$ haviam recebido algum tipo de imunossupressor.

No que se refere ao uso de medicamentos no momento da coleta do exame citológico: $7(9,2 \%)$ usavam metotrexato; $3(3,9 \%)$, ciclofosfamida, $5(6,5 \%)$, azatioprina, $1(1,3 \%)$, mofetil micofenolato, 58 (76,3\%), cloroquina e $29(38,15 \%)$ usavam glicocorticóide (na dose de 5 a $60 \mathrm{mg}$ de prednisona/dia; média de 16,4 $\pm 14,08$, mediana de $10 \mathrm{mg} /$ dia).

Nessa amostra existiam $43,3 \%(\mathrm{n}=33)$ usuárias de anovulatórios; $40,7 \%(\mathrm{n}=31)$ eram tabagistas; $34,2 \%(\mathrm{n}=26)$ com anti-RO; $19,7 \%(\mathrm{n}=15)$ com anti-LA e $21,05 \%$ $(\mathrm{n}=16)$ com anti-DNA positivos. A idade média das pacientes com LES foi de 39,05 \pm 9,7 anos e estas tinham, em média, $1 \pm 1,32$ parceiros sexuais.

O grupo controle foi composto por 80 mulheres saudáveis que realizaram exame preventivo de rotina, com média etária de 36,6 $\pm 11,5$ anos ( $\mathrm{p}=0,35$ em comparação com o grupo de LES; Mann-Whitney), com vida sexual ativa e um número médio de parceiros sexuais de $1 \pm 1,04(\mathrm{p}=0,95$ em comparação com o grupo de LES; Mann-Whitney).

No grupo controle existia 1 paciente $(1,2 \%)$ com lesão LSIL (ou NIC I). No grupo de LES, existiam 7 pacientes $(9,4 \%)$ com o exame citológico alterado, sendo: LSIL (ou NIC I) - 2 pacientes; HSIL (NIC II ou III ou carcinoma in situ) -5 pacientes, dos quais 4 eram NIC II e 1 era NIC III. Em nenhum dos grupos apareceram células de significado indeterminado (ASCUS e AGUS). O estudo comparativo entre presença de Papanicolaou alterado em pacientes com e sem lúpus mostrou $\mathrm{p}=0,03$ (Teste de Fisher) (Figura 1 ).

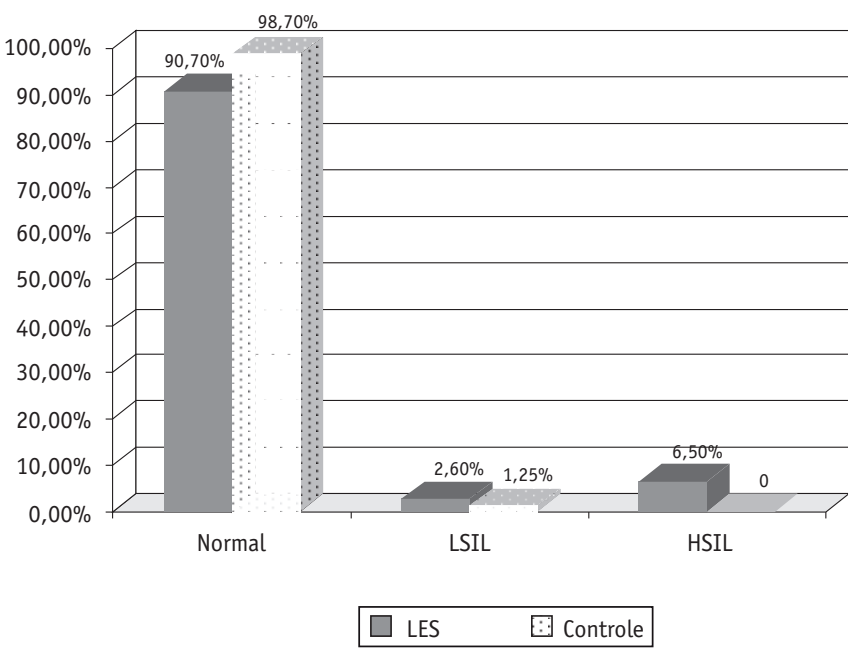

LSIL: lesão intra-epitelial escamosa de baixa malignidade; HSIL: lesão intra-epitelial escamosa de alta malignidade.

Figura 1 - Prevalência de alteração em esfregaço cervicovaginal em pacientes com lúpus eritematoso sistêmico e em controles.

O cálculo do odds ratio (OR) para a prevalência de anormalidades no esfregaço cervicovaginal da amostra de pacientes de LES estudada em relação à população normal foi de 8 (IC de 95\% = 0,9-66). Este cálculo para lesões de baixo grau (LGSIL) demonstra OR de 2,13 (IC de 95\% = $0,18-24)$ e para as lesões de alto risco (HGSIL), um OR = 12,3 (IC de $95 \%=0,67-228$ ).

Os resultados da análise das diferentes variáveis, estudadas nas pacientes com LES, comparando as pacientes com exame citológico alterado e normal são mostrados na tabela 1 .

\section{DISCUSSÃO}

Acredita-se que a ocorrência do câncer de colo de útero está ligada à infecção pelo papilomavírus humano (HPV), uma vez que este vírus tem sido detectado em $90 \%$ das lesões e possui oncogenes com potencial de transformação maligna $^{(10)}$. O HPV é um parasita intracelular capaz de acelerar a velocidade de mitose das células. Isso acontece porque esse vírus, ao se integrar ao cromossomo das células infectadas, causa inibição e eventual degradação do p53 e da proteína $\mathrm{Rb}$, que são elementos supressores e controladores da reprodução celular ${ }^{(10)}$.

Infecções virais podem ser totalmente eliminadas do organismo infectado, podem persistir em formas latentes (com ou sem períodos de replicação) ou, ainda, como infecção crônica ${ }^{(13)}$. As interações entre vírus e o sistema imunológico do hospedeiro são decisivas na evolução do processo 
TABELA 1

Dados COMPARATIVOS NA POPULAÇÃO DE LÚPUS ERITEMATOSO SISTÊMICO (LES) COM E SEM ESFREGAÇO CERVICOVAGINAL ALTERADO

\begin{tabular}{|c|c|c|c|}
\hline & $\begin{array}{c}\text { Citológico } \\
\text { alterado } \\
n=7\end{array}$ & $\begin{array}{c}\text { Citológico } \\
\text { normal } \\
n=69\end{array}$ & $\mathrm{p}$ \\
\hline Média etária (em anos) & $38,8 \pm 9,92$ & $39,9 \pm 9,8$ & $0,89\left(^{*}\right)$ \\
\hline $\begin{array}{l}\text { Tempo médio de doença } \\
\text { (em meses) }\end{array}$ & $90,8 \pm 81,9$ & $75,3 \pm 44,7$ & $0,85\left(^{*}\right)$ \\
\hline Tabagismo & $n=5(71,4 \%)$ & $\mathrm{n}=26(37,8 \%)$ & $0,10(\dagger)$ \\
\hline Anticoncepcionais & $n=3(42,8 \%)$ & $\mathrm{n}=30(43,3 \%)$ & $1,0(\dagger)$ \\
\hline Glomerulonefrite & $\mathrm{n}=0$ & $\mathrm{n}=28(40,5 \%)$ & $0,04(\dagger)$ \\
\hline $\begin{array}{l}\text { Prednisona em mg/dia } \\
\text { (uso atual - dose média) }\end{array}$ & $7,074 \pm 12,2$ & $6,82 \pm 13,8$ & $0,78\left(^{*}\right)$ \\
\hline $\begin{array}{l}\text { Metotrexato } \\
\text { (uso prévio e atual) }\end{array}$ & $\mathrm{n}=0$ & $n=9(13,0 \%)$ & $0,58(\dagger)$ \\
\hline $\begin{array}{l}\text { Ciclofosfamida } \\
\text { (uso prévio e atual) }\end{array}$ & $\mathrm{n}=1(14,2 \%)$ & $\mathrm{n}=17(24,6 \%)$ & $1,0(\dagger)$ \\
\hline $\begin{array}{l}\text { Azatioprina } \\
\text { (uso prévio e atual) }\end{array}$ & $\mathrm{n}=1(14,2 \%)$ & $\mathrm{n}=11(15,9 \%)$ & $1,0(\dagger)$ \\
\hline $\begin{array}{l}\text { Qualquer imunossupressor } \\
\text { (prévio ou atual) }\end{array}$ & $\mathrm{n}=2(28,5 \%)$ & $\mathrm{n}=27(39,1 \%)$ & $0,7(\dagger)$ \\
\hline $\begin{array}{l}\text { Antimaláricos } \\
\text { (uso prévio e atual) }\end{array}$ & $n=3(42,8 \%)$ & $\mathrm{n}=55(79,7 \%)$ & $0,0502(\dagger)$ \\
\hline Anti-Ro & $n=3(42,8 \%)$ & $\mathrm{n}=23(33,3 \%)$ & $0,68(\dagger)$ \\
\hline Anti-La & $\mathrm{n}=2(28,5 \%)$ & $\mathrm{n}=12(17,3 \%)$ & $0,34(\dagger)$ \\
\hline Anti-DNA & $\mathrm{n}=1(14,2 \%)$ & $\mathrm{n}=15(21,2 \%)$ & $1,0(\dagger)$ \\
\hline
\end{tabular}

$\left({ }^{*}\right)$ - Teste de Mann-Whitney.

$(\dagger)$ - Teste de Fisher.

infeccioso e das estratégias para a sua prevenção. Pacientes com LES são imunossuprimidos tanto pela doença básica quanto pelo uso de medicamentos, o que pode prejudicar a capacidade de resistência à infecção pelo HPV. Este é um fato constatado por Berthier et al. ${ }^{(14)} \mathrm{em}$ um trabalho recente, no qual observam que mulheres com LES têm maior prevalência de infecção pelo HPV. Outros autores evidenciaram que as pacientes com LES apresentavam uma maior suscetibilidade tanto para adquirir subtipos do HPV com alto potencial oncogênico como para fazer infecções múltiplas ${ }^{(6)}$. Entre os fatores que prejudicam a capacidade de defesa dos pacientes com LES, estão os defeitos na imunidade celular e no sistema inato de defesa (polimorfismo de genes da via da manana lectina) $)^{(6)}$.

O presente estudo demonstrou uma prevalência aumentada de alterações no exame citológico do colo uterino em pacientes com LES, resultado este que está de acordo com o de outros autores. Em um estudo canadense, Bernatsky et al. ${ }^{(8)}$ evidenciaram uma prevalência de $13,3 \%$ de exames citológicos anormais em pacientes com LES. Também Ognenovski et al..$^{(9)}$, da Universidade de Michigan, Estados Unidos, mostraram uma prevalência de $9,8 \%$ de exames citológicos com alteração em pacientes com LES, contra 3,2\% de exames alterados nos controles. Tam et al ${ }^{(6)}$ encontraram 14 exames de Papanicolaou alterados em 85 pacientes com LES $(16,5 \%)$ comparados com uma prevalência de 5,7\% de alterações no grupo controle. Neste mesmo estudo, as pacientes com LES tinham 5,9 vezes mais chance de apresentar um Papanicolaou com LGSIL e uma chance 7,6 vezes maior de apresentar HGSIL. Na análise atual, a chance de LGSIL foi de 2,1 e a de HGSIL foi de 12,3 na população com LES em relação à normal.

No que diz respeito às variáveis analisadas no presente estudo, dentro do grupo das pacientes com lúpus, comparando-se àquelas que possuíam exame de Papanicolaou alterado com as que não o tinham, não se demonstrou associação entre alteração do exame citológico e tempo de doença, tabagismo, uso de anticoncepcionais, metotrexato, azatioprina, ciclofosfamida, presença de anti-RO, anti-LA e anti-DNA.

Tam et al. ${ }^{(6)}$ relataram que a maior prevalência de exames alterados está relacionada com os fatores de risco inerentes a toda a população, acrescentando que o uso de imunossupressores foi similar nos grupos de pacientes com exames alterados e normais, ou seja, que não houve associação com o seu uso. Todavia, outros autores evidenciaram uma maior prevalência de exames alterados em usuárias de ciclofosfamida e prednisona, sendo tal efeito dependente da dose e do tempo de uso desses medicamentos ${ }^{(9)}$.

Um aspecto interessante observado no presente estudo é a detecção de uma tendência para associação negativa entre uso de antimaláricos e aparecimento de alterações no esfregaço vaginal. Esse é um aspecto que merece maiores estudos principalmente quando se observa que antimaláricos são utilizados para potencializar ação de quimioterápicos em determinadas formas de tumores (como os glioblastomas) ${ }^{(15)}$, sugerindo algum tipo de ação desses medicamentos em células malignas.

Em virtude da maior prevalência de lesões cervicais em mulheres com LES, torna-se mandatório que o reumatologista que acompanha essas pacientes aconselhe a realização periódica de exames para rastreamento de câncer de colo de útero.

No ano de 2006, a Food and Drug Administration (FDA) aprovou a licença de uma vacina ${ }^{(16)}$ recombinante, efetiva contra os subtipos 6, 11, 16 e 18 do HPV e indicada 
para a prevenção do câncer de colo uterino em mulheres com idade entre 9 e 26 anos. Sua eficiência está estimada em $70 \%$, na população normal ${ }^{(15)}$. Caso a vacina se mostre segura e efetiva em mulheres com LES, esta pode vir a ser uma estratégia alternativa a ser utilizada, visando à prevenção do câncer de colo uterino em tais pacientes.

\section{REFERÊNCIAS}

1. Sultan SM, Ioannou Y, Isenberg DA: Is there an association of malignancy with systemic lupus erythematosus? An analysis of 276 patients under long-term review. J Reumatol 39: 1147-52, 2000.

2. Petterson T, Pukkala E, Teppo L, Friman C: Increased risk of cancer in patients with systemic lupus erythematosus. Ann Rheum Dis 51: 437-9, 1992.

3. Ramsey-Goldman R, Mattai SA, Schilling E, et al.: Increased risk of malignancy in pacients with systemic lupus erythematosus. J Invest Med 46: 217-22, 1998.

4. Sweeney DM, Manzi S, Janosky J, et al.: Risk of malignancy in women with systemic lupus erythematosus. J Rheumatol 22: 1478-82, 1995.

5. Abu-Shakra M, Gladman DD, Urowitz MB: Malignancy in systemic lupus erythematosus. Arthritis Rheum 39: 1050-4, 1996.

6. Tam LS, Chan AY, Chan PK, Chang AR, Li EK: Increased prevalence of squamous intraepithelial lesions in systemic lupus erythematosus: association with human papillomavirus infection. Arthritis Rheum 50: 3619-25, 2004.

7. Bernatsky S, Boivin J, Joseph L, et al.: The prevalence of factors influencing cancer risk in lupus: social habits, reproductive issues and obesity. J Rheumatol 29: 2551-4, 2002.

8. Bernatsky S, Ramsey-Goldman R, Gordon C, et al.: factors associated with abnormal pap results in systemic lupus erythematosus. J Rheumatol 43: 1386-9, 2004.
Concluindo, pode-se dizer que o presente estudo mostrou que a população analisada de pacientes com LES tem um risco aumentado de apresentar anormalidades no esfregaço cervicovaginal e que, nesta amostra, a ocorrência dessas anormalidades não depende do uso de imunossupressores, perfil de anticorpos, uso anterior de anovulatórios ou tempo da doença.

9. Ognenovski VM, Marder W, Somers EC, et al.: Increased incidence of cervical intraepithelial neoplasia in women with systemic lupus erythematosus treated with intravenous cyclophosphamide. J Reumatol 31: 1763-7, 2004.

10. Rivoire W, Capp E, Monego H, Appel M, Reis R: Lesões de baixo e alto grau no colo uterino. In: Freitas F, Menke CH, Rivoire W, Passos EP (eds.). Rotinas em ginecologia. 4.ed. Artmed: São Paulo, 2003. pp. 261-72.

11. Tan EM, Cohen AS, Fries JF, et al.: The 1982 revised criteria for the classification of systemic lupus erythematosus. Arthritis Rheum 25: 1272-7, 1982.

12. The 1988 Bethesda System for reporting cervical/vaginal cytological diagnosis. National Cancer Institute Workshop. JAMA 262: 931, 1989.

13. Roitt I, Brostoff J, Male D: Immunity to viruses. In: Roitt I, Brostoff J, Male D (eds.). Immunology, 5.ed. Mosby: London, 1998. pp. 221-8.

14. Berthier S, Mougin C, Vercherin P, et al.: Does a particular risk associated with papillomavirus infections exist in women with lupus? Rev Med Intern 20: 128-32, 1999.

15. Savarino A, Lucia MB, Giordano F, Cauda R: Risks and benefits of chloroquine use in anticancer strategies. Capturado em http://oncology.thelancet.com vol. 7: 792-3, 2006.

16. Product Approval Information. Gardasil ${ }^{\circledR}$. U.S. Food and Drug Administration (FDA), capturado em http://www.fda.gov/ cber/label/hpvmer060806LB.htm em 01/2007. 